\title{
Chromophore arrangement in light-harvesting complex II influenced by the protein dynamics on the microsecond time scale
}

\author{
Sebastian Thallmair ${ }^{1, *}$, and Siewert J. Marrink ${ }^{1}$ \\ ${ }^{1}$ Groningen Biomolecular Sciences and Biotechnology Institute and The Zernike Institute for \\ Advanced Materials, University of Groningen, Nijenborgh 7, 9747 AG Groningen, The Netherlands
}

\begin{abstract}
Coarse-grained molecular dynamics simulations of lightharvesting complex II in thylakoid membrane reveal its microsecond dynamics. We analyze the fluctuations of the relative chromophore orientations which set the stage for the energy transfer.
\end{abstract}

\section{Antenna protein light-harvesting complex II}

Plant chloroplasts contain considerable amounts of the antenna protein light-harvesting complex II (LHCII) which is a key player in natural photosynthesis. It is associated with the photosystem II (PSII) and occurs as a trimer. Each monomer contains a variety of different cofactors: 8 chlorophyll a, 6 chlorophyll b, and 4 carotenoid molecules. They are responsible for capturing photons and transmitting the excitation energy towards the PSII reaction center. This challenging task requires a highly precise arrangement of the involved chromophores resulting in a specifically fine-tuned ordering of the energy levels and chromophore couplings.

\section{Long term fluctuations set the stage for excitation energy transfer}

The delocalization and transfer of excitation energy in antenna proteins takes place on the picosecond time scale. In contrast, the dynamics of the protein matrix in which the optically active chromophores are embedded covers a much longer time scale. This implies that the fluctuations in the protein do not drastically change one single excitation event but they generate a manifold of different conditions in terms of chromophore distances and orientations which are all present at the same time in chloroplasts. Thus, the antennae proteins have to deal with all these different predefined boundary conditions in order to efficiently funnel the excitations towards the PSII reaction center.

* Corresponding author: s.thallmair@rug.nl 


\section{Coarse-grained molecular dynamics model}

We built a coarse-grained (CG) model of LHCII using the Martini force field [1]. In this force field, about four non-hydrogen atoms are grouped into one interaction site preserving the chemical nature of the interaction site. This allows exploring the dynamics of large biomolecular ensembles on the $\mu$ s time scale. To model specific hydrogen bonds which are particularly important to maintain the secondary structure of the protein, we employ a Gō model [2]. The coordinate covalent bond between the central $\mathrm{Mg}^{2+}$ ions of the chlorophylls and the protein are added explicitly. As a benchmark of our newly developed LHCII model serve recent atomistic molecular dynamics data [3] (Fig. 1, left). As the CG model allows for an about three orders of magnitude faster sampling, we are now able to study the dynamics of LHCII monomers and trimers on a hundreds of $\mu$ s time scale. While doing so, we place the antenna proteins in their natural environment - the thylakoid membrane.
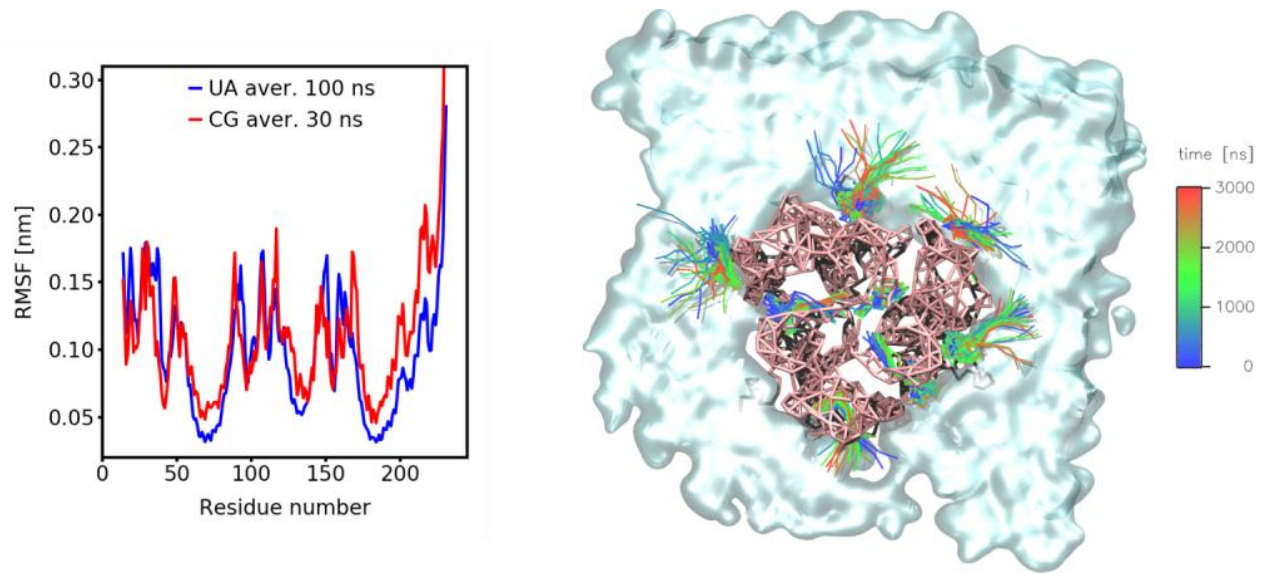

Fig. 1. Comparison of the root mean square fluctuations (RMSF) of our new GC model for the LHCII monomer (red line) with atomistic simulations (blue line; left panel). Top view of the LHCII trimer embedded in thylakoid membrane (right). The protein backbone is colored pink; the membrane is drawn as cyan surface. Eight selected chlorophyll molecules are depicted over the whole simulation time of $3 \mu$ s to show the differences of their flexibility. Each chlorophyll molecule is depicted every $125 \mathrm{~ns}$. The color bar indicates the corresponding simulation time.

\section{Dynamics at the coarse-grained level}

As depicted in Fig. 1 (left), the root mean square fluctuations of the protein backbone in the CG model show a good agreement with the atomistic data demonstrating that we are able to study protein and cofactor flexibility. The simulations of the LHCII trimer in thylakoid membrane reveal the high flexibility of the aliphatic tails of the chlorophylls at the outer surface of the protein. In contrast, the tails of the chlorophylls located at the protein-protein interfaces are much more static. Fig. 1 (right) shows the protein backbone network (pink) in its configuration at $\mathrm{t}=0 \mu \mathrm{s}$ while the temporal evolution of eight selected chlorophyll molecules is indicated using the color bar. The behavior of LHCII for $3 \mu \mathrm{s}$ in total is depicted. The higher flexibility of the chlorophyll tails at the interface with the membrane is due to their interactions with the lipid tails. The thylakoid membrane contains a large amount of glycolipids with highly flexible unsaturated lipid tails promoting the structural disorder of the chlorophyll tails. 


\section{Exploration of the orientation space of the chromophores}

To evaluate the flexibility of the chlorophylls and to which extent they explore their orientation space, we calculated the distances of the central $\mathrm{Mg}^{2+}$ ions as well as the angles between the aromatic moieties. As an example, we selected chlorophyll a 604 (red, Fig. 2) and evaluated the orientation to four other chlorophylls in the same LHCII monomer. Overall, the chlorophylls show to be relatively stable and maintain at their residues coordinating the central $\mathrm{Mg}^{2+}$ ion in the crystal structure. They mainly explore the vicinity of their initial position resulting in a standard deviation of the distance fluctuations observed in our CG trajectories on the order of a few $\AA$ (see Fig. 2, middle). The right panel of Fig. 2 illustrates the time evolution of the angle between the normal vectors of the planar aromatic rings of the chlorophylls. While for example the angle between cla604 and clb601 (green line) is relatively stable fluctuating on the order of 10-50 ns, the orientation of cla604 and cla613 fluctuates on the $\mu$ s time scale by about $30^{\circ}$.
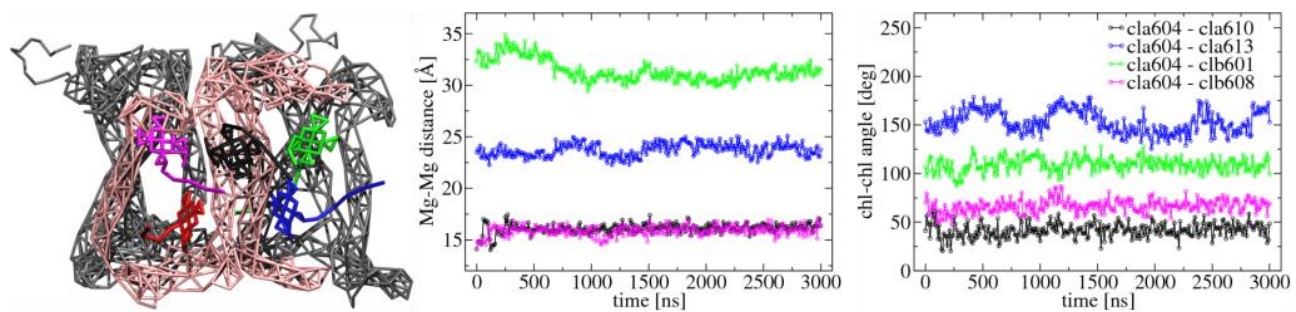

Fig. 2. Side view of the LHCII trimer (left). The protein backbone of the monomer which embeds the depicted chlorophylls is colored pink; the other two gray. Chlorophyll a 604 is colored in red; chlorophyll a 610 black; chlorophyll a 613 blue; chlorophyll b 601 green; chlorophyll b 608 magenta. In addition, the $\mathrm{Mg}-\mathrm{Mg}$ distances (middle) and the angles between the aromatic rings (right) between chlorophyll a 604 (red) and the other chlorophylls are depicted. The lines are colored correspondingly.

\section{Summary}

In summary, our simulations shine light on the diverse variety of chromophore arrangements in the trimeric antenna complex LHCII and can provide access to the corresponding manifold of electronic conditions. Although the excitation transfer occurs on a much shorter time scale than the observed fluctuations, the latter predefine the electronic conditions which the excitation encounters in the antenna complex.

This work was supported by the European Commission via a Marie Skłodowska-Curie Actions Individual Fellowship to S.T. (MicroMod-PSII, grant agreement 748895).

\section{References}

1. S. J. Marrink, H. J. Risselada, S. Yefimov, D. P. Tieleman, A. H. de Vries, J. Phys. Chem. B 111, 7812 (2007)

2. A. B. Poma, M. Cieplak, P. E. Theodorakis, J. Chem. Theory Comput. 13, 1366 (2017)

3. N. Liguori, X. Periole, S. J. Marrink, R. Croce, Sci. Rep. 5, 15661 (2015) 